Alambiaga I, Álvarez E, Diez-Méndez D, Verdejo J and Barba E. "The tale of the three little tits": different nest building solutions under the same environmental pressures. Avian Biol Res 2020; 13(3): 49-56. https://doi.org/10.1177/1758155920943116

\title{
"The tale of the three little tits": different nest building solutions under the same environmental pressures
}

\section{Iván Alambiaga ${ }^{1}$, Elena Álvarez ${ }^{1}$, David Diez-Méndez ${ }^{2,3}$, José Verdejo ${ }^{4}$ and Emilio Barba ${ }^{1}$}

${ }^{1}$ Cavanilles Institute of Biodiversity and Evolutionary Biology, University of Valencia, Paterna, Spain.

${ }^{2}$ National Museum of Natural Sciences (CSIC), C/José Gutiérrez Abascal 2, E-28006 Madrid, Spain. ${ }^{3}$ Institute of Entomology, Biology Centre, Czech Academy of Sciences, Branišovská 31, 37005 České Budějovice, Czech Republic.

${ }^{4}$ Microbiology and Ecology Department University of Valencia, c/Doctor Moliner 50, 46100 Burjassot, Spain.

ORCID: IA: 0000-0001-7951-5207; D.D.-M.: 0000-0003-0218-3421; EB: 0000-0003-2882-9788

Corresponding author:

Emilio Barba, Cavanilles Institute of Biodiversity and Evolutionary Biology, University of Valencia, c/Catedrático José Beltrán 2, 46980 Paterna, Spain.

Email: Emilio.barba@uv.es

\section{Abstract}

Evolutionary selection pressures, and species-specific ecology and behaviour, promote a great variability in the size and composition of nests. However, it would be expected that phylogenetically close species, with similar ecological needs, breeding at the same time in the same place, would also build similar nests. In contrast with this, previous studies have found differences in nest mass and composition among closely related sympatric species. These differences have been attributed to small differences in body size (smaller species building larger and/or more insulated nests), or to the different ways in which species perceive the environment (e.g. perceived predation risk). In this study, for the first time, we searched for differences between nest mass, composition, and importance of the different functional parts of the nest between Blue (Cyanistes caeruleus), Great (Parus major) and Coal tits (Periparus ater) breeding under the same conditions. We found that smaller species built larger nests and/or include more thermoregulatory materials, probably having greater insulating capacity, which agrees with previous hypotheses. In particular, Blue Tits made greater use of bark, feathers and vegetable fibre, while Great Tits used wild boar hair in greater proportions. In addition, for the first time, we described in detail the nest composition of Coal Tits, which contained large amounts of fluff compared to the other two species. All these results are in line with previous hypothesis linking nest size and composition to the size of the birds, and the existence of species-specific characteristics in the selection of materials for nest building. 
37 Key words: Cyanistes caeruleus, Parus major, Periparus ater, mixed forest, nest size, nest insulation, thermoregulation, structuring materials.

40 Introduction

41 Most birds build nests in order to have a suitable environment in which their embryos and 42 hatched offspring can develop. ${ }^{1}$ Nests have a wide taxonomical distribution, and their design and 43 location vary markedly between and within taxa according to phylogeny, environmental 44 conditions, predation pressure, parasitism, or sexual selection., ${ }^{2,3}$ Temperature experienced 45 during nest construction, for example, has been observed to influence the design of nests, 46 resulting in better insulated nests being built when temperatures are colder. ${ }^{4-6}$ Nest 47 characteristics, and the behaviour associated to its building, affect the fitness of the breeding 48 individuals through several pathways. ${ }^{1,7,8}$ For example, positive correlations between nest size 49 and clutch size, ${ }^{9}$ or hatching and breeding success, ${ }^{10,11}$ have been found.

50 Different bird species might use different types of materials to achieve the same functions, while 51 the same materials could serve as different, simultaneous, function in the same nest., ${ }^{1,3}$ For 52 example, moss, a main structural material for tit (Paridae) species, has also an important 53 thermoregulatory function, ${ }^{12}$ while feathers, used mainly for their insulation properties, could 54 also serve as "decorative" materials, signalling the quality of the male bringing them. ${ }^{13}$ The type 55 of materials used to build the nest, and the amount and proportion in which they are used, can 56 also affect reproductive parameters. ${ }^{3,14,15}$ The quantity of moss and its proportion in the total nest 57 mass of Great Tits (Parus major), for instance, has been proved to have a positive effect on 58 hatching and fledging success. ${ }^{16}$

59 Intraspecific variation in nest size and design due a large number of factors such as nestbox size, 60 habitat type, availability of food and construction materials, predation risk, parasitism, or the 61 quality of the builders on nest size and design has been extensively explored. ${ }^{3,11,17-20}$ However, 62 nest design comparisons between closely related sympatric species are less frequent Based on the previously mentioned factors known to affect nest design, it would be expected that phylogenetically close species, ${ }^{21}$ with equivalent ecological niches, and breeding by the same time in the same habitat, would build similar nests. However, consistent variation has been found between species under these conditions, and these have been attributed to differences in body size, ${ }^{22}$ nest building behaviour, ${ }^{23}$ differential use of available resources, ${ }^{24}$ or environment perception, for example, that the same objective risk of predation might be perceived differently by species of different size. 22,25

We aimed to assess differences in nest characteristics among three closely related sympatric species of the Paridae family: the Great Tit, the Coal Tit (Periparus ater) and the Blue Tit (Cyanistes caeruleus). Previous studies comparing the nest size of Great and Blue tits under standardized conditions have found discordant results, some of them showing that both species build nests of similar size and mass, ${ }^{24,25}$ while others conclude that Great Tits build smaller and lighter nests than Blue Tits. ${ }^{22,26-29}$ On the other hand, consistent differences have been found between the two species in the proportion in which different nest materials appeared in the nests. ${ }^{24,29,30}$ Thus, Blue Tits generally make a greater use of bark and feathers, while Great Tits use relatively fewer feathers and more hair. Comparisons of nest characteristics involving other Paridae species are, however, exceptional. To the best of our knowledge, the only direct comparison between nests of Coal, Blue and Great tits was made by Lambrechts et al. ${ }^{25}$ That study concluded that the 
external height of the nest walls, the only parameter actually measured, was larger in Coal Tits than in Great and Blue tits when co-existing in the same coniferous woodland. It should be also stressed that, contrasting with the several detailed description of Great and Blue Tit nests, ${ }^{31}$ we are not aware of any one reporting nest mass and composition of Coal Tits, so we will describe them here for the first time.

Variation in nest size and composition between sympatric tit species has been suggested to be generally caused by differences in the way that each species perceives its environment. ${ }^{22,25}$ For example, small differences in body size might make smaller species to be more sensitive to cold temperatures, so they would be expected to build larger and more insulated nests. ${ }^{22}$ Also, the need to keep a minimum security distance between the nest content (especially nestlings and incubating females) and the entrance of the nestbox, to reduce predation risk, might press bigger species to build shorter nests. ${ }^{27,28}$

In this study, we searched for specific differences in mass and composition of the nests of Great, Blue and Coal tits, breeding at the same time in the same habitat, and using the same nestbox type and size. Our predictions, based on the above-mentioned studies on these three species, and others using different species, ${ }^{32,33}$ are that (1) smaller species will build heavier nests than larger ones, (2) smaller species will use a higher proportion of insulating materials, and (3) the use of nest materials would be species-specific, following, for Great and Blue tits, the patterns found in previous studies. ${ }^{24,34,35}$

\section{Methods}

The study was performed in Monte de Santa Bárbara de Pina (Castellón, Spain, 40 01' N, $0^{\circ} 38^{\prime}$ W, 1200 m a.s.I.), during three breeding seasons (2016, 2017 and 2018). The area was a mainly Maritime Pine (Pinus pinaster Aiton, 1789) forest, with scattered, and generally smaller in size, Portuguese Oaks (Quercus faginea Lam., 1785). As the two species are not distributed in patches, but are interspersed forming a homogeneous landscape, we believe that there is little variation in environmental conditions, including temperature and humidity, parasite pressures, predation risk, or availability of food and nest-building materials, throughout the study area. We recorded, for other study, temperatures within some occupied nestboxes during the laying period (Thermochron iButton data loggers, accuracy $\pm 0.5^{\circ} \mathrm{C}$, Model DS1922L-F5, Maxim Integrated, CA, USA), so they could serve as an approximation of general thermal conditions around the time of nest construction. Mean minimum recorded temperatures were $3.3 \pm 2.5^{\circ} \mathrm{C}$ ( $n=11$ nests) in 2016, $-0.7 \pm 2.3^{\circ} \mathrm{C}(n=6)$ in 2017 , and $3.1 \pm 2.4^{\circ} \mathrm{C}(n=10)$ in 2018. Potential nest predators in this area include Red Squirrels (Sciurus vulgaris), Stone Martens (Martes foina), Common Genet (Genetta genetta), Montpellier (Malpolon monspessulanus) and Ladder (Zamenis scalaris) snakes, and Great Spotted (Dendrocopos major) and European Green (Picus viridis) woodpeckers.

Wooden nestboxes were distributed along trails within the study area, and about $50 \mathrm{~m}$ away from each other. They had a front removable door with a circular entrance hole of $3.2 \mathrm{~cm}$ diameter. The wall thickness was $1.5 \mathrm{~cm}$, the bottom area (inside) had $11.6 \times 10.6 \mathrm{~cm}$, and the distance from the floor to the lower edge of the entrance hole was $10.5 \mathrm{~cm}$. Less than $20 \%$ of the nestboxes were occupied in any particular year, so we consider that they were in excess for the needs of local hole-nesting species. Each nestbox was visited at least once a week from March to June, to follow the breeding process of the three studied species. 
124 Females build the nest in the three species, ${ }^{34}$ so the three species would have the same time and energetic constraints during nest-building. Laying date of the first egg (assuming one egg was laid per day) and clutch size were recorded for each nest. Considering the nests included in the present study, we did not find differences between species in any of the two parameters (laying date: $F_{2,44}=0.88, P=0.42$; clutch size: $F_{2,44}=0.48, P=0.63$ ). Females were captured and weighed when feeding 10-12 (for Great Tits) or 9-11 days old nestlings (for Blue and Coal tits) using doortraps. Coal Tits were the lightest species (mean $\pm S D=8.61 \pm 0.40 \mathrm{~g}, \mathrm{n}=13$ ), followed by Blue Tits $(10.22 \pm 0.46 \mathrm{~g}, \mathrm{n}=10)$ and Great Tits $(16.97 \pm 0.61 \mathrm{~g}, \mathrm{n}=15)$, each species differing from the other two ones $\left(F_{2,35}=1054.4, P<0.001\right.$, followed by a posteriori Scheffe pairwise comparisons). Finally, once fledglings left the nestbox, nests were removed and stored individually in plastic bags at $-20^{\circ} \mathrm{C}$. A total of $15 \mathrm{Great} \mathrm{Tit,} 10$ Blue Tit and 15 Coal Tit nests, where at least one chick fledged, were collected (15 in 2016, 12 in 2017 and 13 in 2018). As far as we know (not all the females were captured), most nests included in this sample were built by different females: only 3 Great Tit, 3 Coal Tit, and one Blue Tit females are known to have contributed 2 nests each.

All the collected nests were deconstructed by the same person (IA), and the different materials (moss, fluff, bark, vegetal fibre, sticks, feathers, and wild boar hair) were dried independently during 12 hours at $105^{\circ} \mathrm{C}$, following the same protocol as Álvarez and Barba ${ }^{36}$. Each component was weighed using an electronic balance with an accuracy of $0.01 \mathrm{~g}$. Components weighing less than that, which included some leaves, were not taken into account. The dry mass of each nest was the sum of all its component parts.

Components were also classified into two functional groups ("structuring" and "thermoregulatory" materials) to test for differences in the importance of the distinct functional parts of the nest. Since some materials could have various functions, ${ }^{3}$ we have classified each material considering what is considered to be the main one. Thus, components that would have been mainly used to compact and stabilize the structure (moss, vegetal fibre, bark, sticks, and rigid long hair from wild boars) were grouped under "structuring" materials. Fluff, including woolly materials from animal and artificial sources, and feathers, were put together under the "thermoregulatory" material group, as their insulating properties are well known, ${ }^{37-39}$ and were mostly used to line the nest cup.

To assess differences among the masses of each component, the two functional groups, and total nest mass between species, we built a linear model (LM) for each variable of interest with an identity link (Gaussian family) for each variable of interest. We used species as an explanatory categorical variable and also considered year as factor to account for the variability between breeding seasons. Residuals of the response variable were visually inspected to check for normality and homogeneity of the variance. Due to a small sample size, we corrected LMs estimates when we found data points showing high leverage, or outliers, using the Robustbase package. ${ }^{40}$ Applying this correction, we avoided highly influential data points by assigning them lower weights in the LMs. Residuals in the model analysing the weight of wild boar hair showed heteroscedasticity; hence, estimates were corrected using the Sandwich package. ${ }^{41}$ We reported model $R^{2}$ values and considered significant $p$-values lower than 0.05 . All the analyses were conducted using R 3.5.2. ${ }^{42}$ 
168

169

\begin{tabular}{llll}
\hline & Great Tit & Blue Tit & Coal Tit \\
& $\mathrm{n}=15$ nests & $\mathrm{n}=10$ nests & $\mathrm{n}=15$ nests \\
\hline Components & Mean \pm SD & Mean \pm SD & Mean \pm SD \\
\hline Moss & $9.29 \pm 3.77$ & $8.89 \pm 2.33$ & $10.51 \pm 3.64$ \\
Fluff & $1.29 \pm 1.06$ & $0.76 \pm 0.70$ & $3.18 \pm 1.62$ \\
Bark & $0.15 \pm 0.28$ & $2.33 \pm 1.76$ & $1.08 \pm 1.86$ \\
Wild boar hair & $0.30 \pm 0.29$ & $0.07 \pm 0.15$ & $0.02 \pm 0.06$ \\
Feathers & $0.13 \pm 0.24$ & $0.39 \pm 0.33$ & $0.09 \pm 0.13$ \\
Vegetal fibre & $0.21 \pm 0.20$ & $0.37 \pm 0.25$ & $0.19 \pm 0.18$ \\
Sticks & $0.45 \pm 1.18$ & $0.09 \pm 0.13$ & $0.37 \pm 0.49$ \\
\hline Functional groups & & & \\
\hline Structuring & $10.39 \pm 4.33$ & $11.75 \pm 2.93$ & $12.17 \pm 3.16$ \\
Thermoregulation & $1.42 \pm 1.13$ & $1.15 \pm 0.78$ & $3.27 \pm 1.65$ \\
\hline Total mass & $11.81 \pm 4.29$ & $12.90 \pm 3.21$ & $15.44 \pm 3.93$ \\
\hline
\end{tabular}


190

191

192

193

194

195

90

91

2

93

Table 2. Results of the linear models analysing the effect of species and year on the dry mass (g) of nest materials. The categorical variables "species" and "year" were assessed in comparison with the reference species Great Tit and the year 2016.

\begin{tabular}{|c|c|c|c|c|c|}
\hline & & Estimate & SE & $\mathrm{t}$ & $\mathrm{P}$ \\
\hline \multirow{5}{*}{$\begin{array}{l}\text { Fluff } \\
R^{2}=0.41\end{array}$} & Intercept & 1.70 & 0.38 & 4.53 & \\
\hline & Blue Tit & -0.40 & 0.50 & -0.80 & 0.432 \\
\hline & Coal Tit & 1.86 & 0.44 & 4.24 & $<0.001$ \\
\hline & Year 2017 & -0.34 & 0.47 & -0.71 & 0.482 \\
\hline & Year 2018 & -1.02 & 0.46 & -2.23 & 0.033 \\
\hline \multirow{5}{*}{$\begin{array}{l}\text { Bark } \\
R^{2}=0.61\end{array}$} & Intercept & -0.13 & 0.14 & -0.94 & \\
\hline & Blue Tit & 1.54 & 0.21 & 7.40 & $<0.001$ \\
\hline & Coal Tit & 0.27 & 0.17 & 1.61 & 0.116 \\
\hline & Year 2017 & -0.21 & 0.19 & -1.12 & 0.268 \\
\hline & Year 2018 & 0.16 & 0.18 & 0.86 & 0.397 \\
\hline \multirow[t]{5}{*}{$\begin{array}{l}\text { Wild boar hair } \\
R^{2}=0.27\end{array}$} & Intercept & 0.32 & 0.07 & 4.42 & \\
\hline & Blue Tit & -0.21 & 0.10 & -2.08 & 0.045 \\
\hline & Coal Tit & -0.27 & 0.08 & -3.40 & 0.002 \\
\hline & Year 2017 & -0.10 & 0.06 & -1.60 & 0.119 \\
\hline & Year 2018 & -0.01 & 0.10 & -0.06 & 0.956 \\
\hline \multirow{5}{*}{$\begin{array}{l}\text { Feathers } \\
R^{2}=0.34\end{array}$} & Intercept & 0.15 & 0.05 & 3.22 & \\
\hline & Blue Tit & 0.25 & 0.06 & 4.03 & $<0.001$ \\
\hline & Coal Tit & 0.03 & 0.05 & 0.68 & 0.502 \\
\hline & Year 2017 & -0.20 & 0.06 & -3.50 & 0.001 \\
\hline & Year 2018 & -0.08 & 0.05 & -1.57 & 0.126 \\
\hline \multirow{5}{*}{$\begin{array}{l}\text { Vegetal fibre } \\
R^{2}=0.06\end{array}$} & Intercept & 0.19 & 0.06 & 3.31 & \\
\hline & Blue Tit & 0.16 & 0.07 & 2.15 & 0.038 \\
\hline & Coal Tit & -0.01 & 0.06 & -0.16 & 0.871 \\
\hline & Year 2017 & 0.02 & 0.07 & 0.29 & 0.775 \\
\hline & Year 2018 & -0.04 & 0.07 & -0.60 & 0.554 \\
\hline \multirow[t]{5}{*}{$\begin{array}{l}\text { Sticks } \\
R^{2}=0.02\end{array}$} & Intercept & 0.13 & 0.04 & 3.18 & \\
\hline & Blue Tit & -0.05 & 0.06 & -0.88 & 0.380 \\
\hline & Coal Tit & 0.07 & 0.05 & 1.30 & 0.201 \\
\hline & Year 2017 & -0.02 & 0.05 & -0.44 & 0.666 \\
\hline & Year 2018 & 0.00 & 0.06 & 0.04 & 0.969 \\
\hline \multirow{5}{*}{$\begin{array}{l}\text { Moss } \\
R^{2}=0.03\end{array}$} & Intercept & 8.91 & 1.01 & 8.78 & \\
\hline & Blue Tit & -0.03 & 1.35 & -0.03 & 0.980 \\
\hline & Coal Tit & 1.49 & 1.20 & 1.25 & 0.221 \\
\hline & Year 2017 & -1.26 & 1.28 & -0.98 & 0.332 \\
\hline & Year 2018 & 1.30 & 1.25 & 1.04 & 0.306 \\
\hline
\end{tabular}


a)
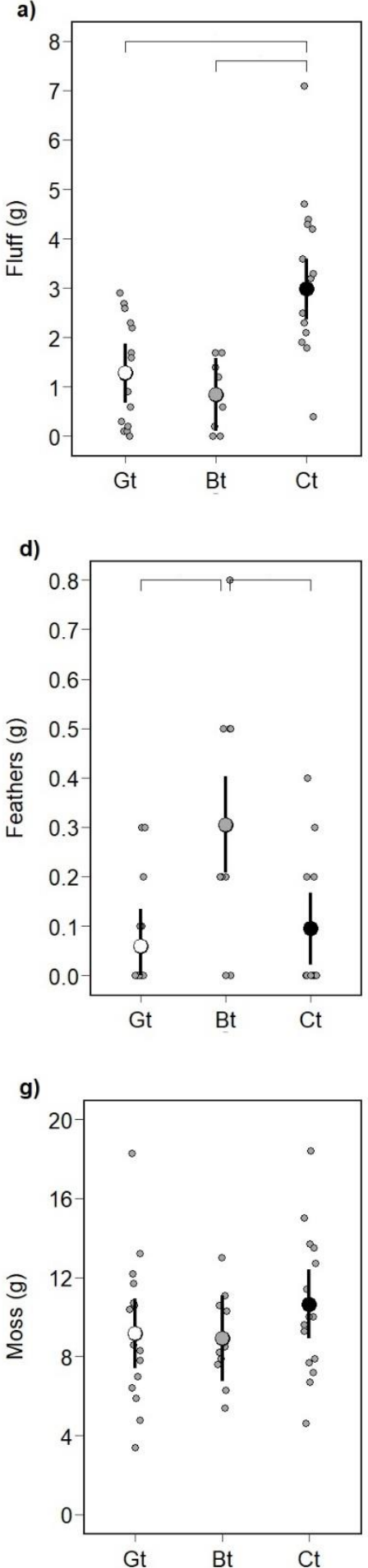

b)

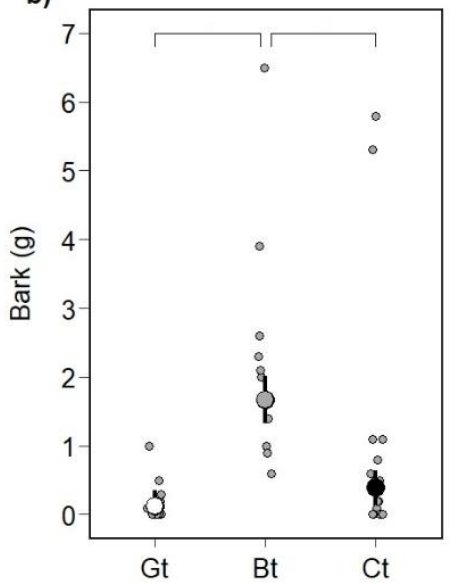

e)

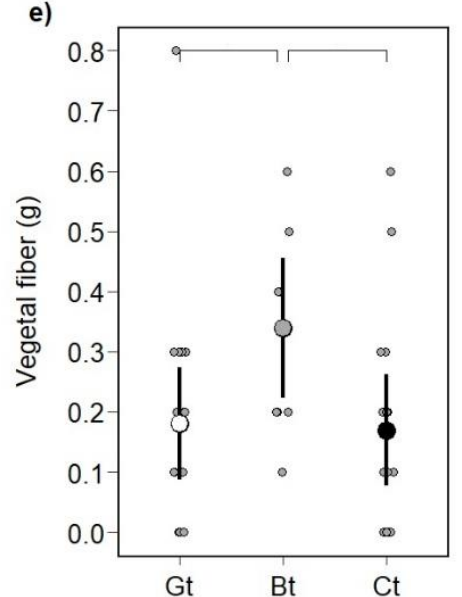

h)

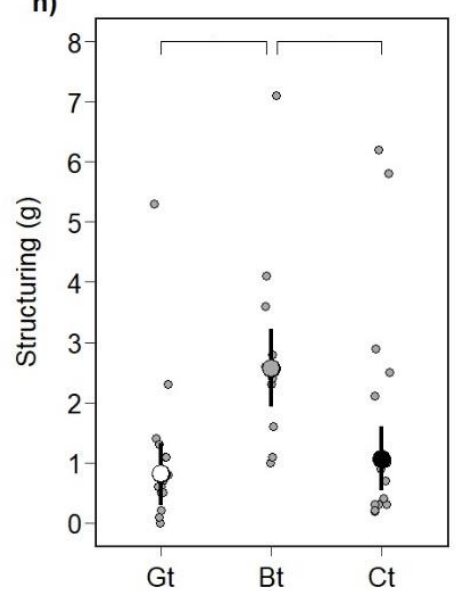

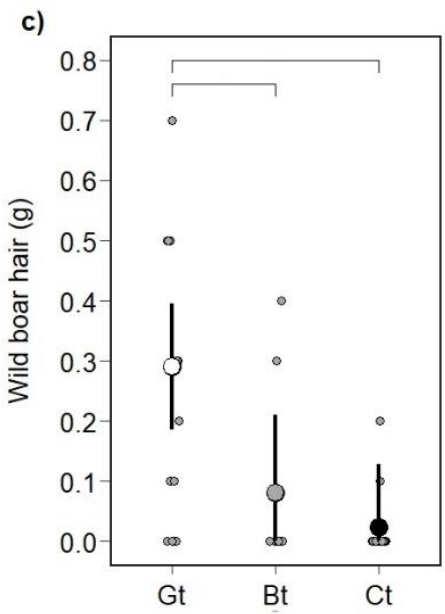

f)

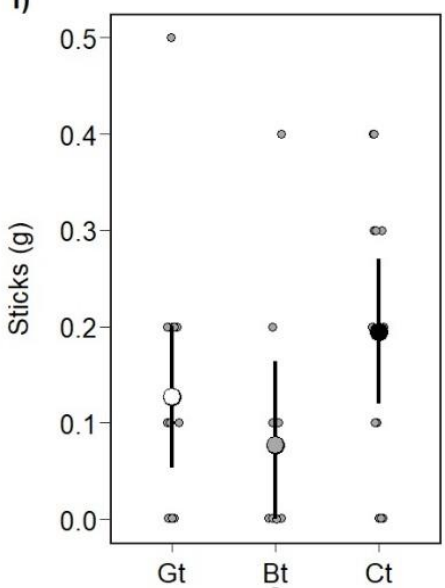

i)

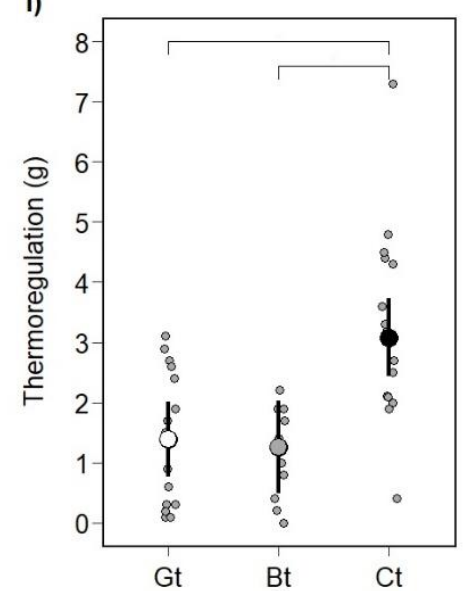

Figure 1. Dry mass (g) of the different nest materials, and the two functional groups in which nest 198 materials were classified, and total nest mass, of the three species. Figures show the mean value 199 and the 95\% interval (black bars) obtained from the resulting linear models with the effects 200 package. ${ }^{43}$ Grey points show raw data for each species. Square brackets indicate statistical differences between species: Gt (Great Tit), Bt (Blue Tit) and Ct (Coal Tit). 
203

204

205

206

207

208

209

210

211

212

213

214

215

216

217

218

219

220

221

222

223

Concerning the two functional groups of materials, Coal Tits added more thermoregulatory materials than the other species, while the amount of components classified under the structuring category did not differ between species (Table 1, Table 3, Figure 1).

Table 3. Results of the linear models analysing the effect of species and breeding year on the dry mass (g) of the different functional groups, in which nest materials were classified, and the total nest weight. The categorical variables "species" and "year" were assessed in comparison with the reference species Great Tit and the year 2016.

\begin{tabular}{llrrrr} 
& & Estimate & SE & \multicolumn{1}{c}{ t } & P \\
\hline Thermoregulatory & & & & & \\
$\mathrm{R}^{2}=0.36$ & Intercept & 1.81 & 0.37 & 4.84 & \\
& Blue Tit & -0.13 & 0.50 & -0.27 & 0.788 \\
& Coal Tit & 1.69 & 0.44 & 3.81 & $<0.001$ \\
& Year 2017 & -0.36 & 0.48 & -0.76 & 0.452 \\
& Year 2018 & -0.96 & 0.46 & -2.08 & 0.045 \\
Structuring & & & & & \\
$\mathrm{R}^{2}=0.12$ & Intercept & 10.14 & 1.06 & 9.57 & \\
& Blue Tit & 1.61 & 1.41 & 1.14 & 0.263 \\
& Coal Tit & 2.15 & 1.24 & 1.73 & 0.093 \\
& Year 2017 & -1.82 & 1.34 & -1.36 & 0.182 \\
& Year 2018 & 1.83 & 1.30 & 1.41 & 0.167 \\
\hline \multirow{2}{*}{ Total nest weight } & & & & & \\
$\mathrm{R}^{2}=0.17$ & Intercept & -11.69 & 1.03 & 11.33 & \\
& Blue Tit & 1.91 & 1.37 & 1.40 & 0.171 \\
& Coal Tit & 3.93 & 1.22 & 3.22 & 0.003 \\
& Year 2017 & -2.09 & 1.29 & -1.62 & 0.115 \\
& Year 2018 & 0.44 & 1.28 & 0.34 & 0.736
\end{tabular}

Finally, total nest mass also differed between species (Table 1, Table 3). Nest mass was negatively related to body size, with Coal Tits, the smallest species, building considerably larger nests than Great Tits, the largest one, with Blue Tits having an intermediate mass and not differing from any of the other two species (Figure 2). 


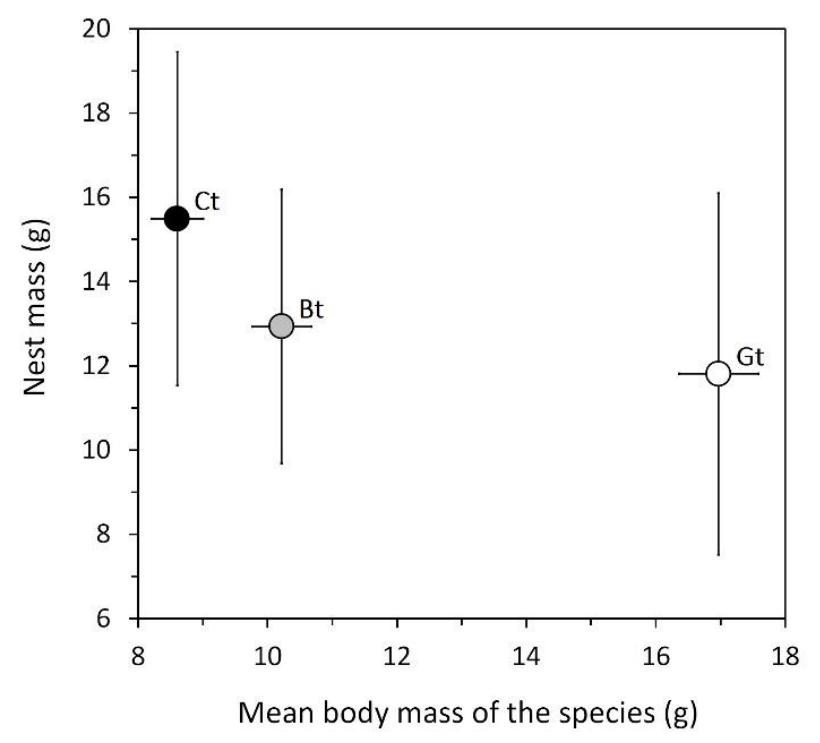

Figure 2. Relationship between the total nest mass and the mean body mass of the three species. Vertical bars show the standard deviation of the nest mass, and the horizontal bars show the standard deviation of the body mass of each species: Gt (Great Tit), Bt (Blue Tit) and Ct (Coal Tit).

\section{Discussion}

232 Our results showed the existence of species-specific characteristics in the nest design of the studied species. Differences were found in total mass, composition, and importance of the different functional parts of the nest between these three similar species when coexisting under the same environmental conditions. These differences could not be attributed to differences in laying dates (a proxy of experienced ambient temperature, and of availability of food and, perhaps, some nest materials as moss) or clutch size between species in our study area. On the other hand, competition between tit species for breeding sites is a well-documented phenomenon, ${ }^{44}$ which could result on the displacement of competitively inferior species to poorer quality habitats. However, the large availability of nestboxes (less than $20 \%$ occupied any particular year), makes it unlikely that competition for particular nestboxes, otherwise placed in a virtually homogeneous habitat, was a relevant issue to consider. Moreover, tits have been observed to move relatively long distances in search of nest building materials, ${ }^{45}$ so, in any case, slight spatial variations affecting the availability of materials should be compensated for by the high mobility of the birds.

246 Most studies comparing species of the Paridae family, virtually all of them only involving Great 247 and Blue tits, have found an inverse relationship between female body size and nest size, ${ }^{27,28,29}$ 248 although some have failed to detect this trend. ${ }^{24,25}$ The main hypothesis to explain this variation is that larger nests, with better insulating properties, ${ }^{46}$ would compensate the vulnerability of smaller species to cold temperatures. ${ }^{22}$ In our study, Coal Tits, the smallest species, built heavier nests than Great Tits, the largest one. The mass of Blue Tit nests did not differ from those of Coal and Great tits, showing an intermediate mass. Thus, our results broadly support the hypothesis that smaller species build larger nests than bigger ones, and the relationship between nest mass and insulation is one of the possible reasons for this trend. 
Predation risk is another reason proposed to explain this relationship between nest size and body size. To keep a similar distance between the top of the nest content (i.e. eggs, nestlings, or female), and the entrance hole, the nest should be shallower when the total volume of the content (the body size of the species is a proxy) is larger. ${ }^{27,35}$ For this reason, Lambrechts et al. ${ }^{28}$ proposed that larger species would need larger safety distances between the top of the nest and the entrance hole, resulting in shallower nests than those of smaller species when breeding in nestboxes of a similar size. In the previous study comparing the same three species, Coal Tits built the tallest nests, but no differences were found between Blue and Great tits, despite the smaller size of the former. ${ }^{25}$ As said above, our results show that mean nest mass increased as body size of the species decreased, which agrees with the predation-avoidance hypothesis. Perhaps, the strength of the differences in nest size between species which could be attributed to predation risk would depend on the specific predators, and their actual pressure over tit nests, at each location. Increasing the "safety distance" could be effective for predators as medium-sized mammals, such as Stone Martens and Common Genets, which capture nestlings by inserting their leg through the entrance hole. ${ }^{27}$ It would be virtually irrelevant, however, for small mammals or snakes, which enter into the nestbox to prey upon eggs, nestlings or females, ${ }^{47,48}$ or for woodpeckers, which enlarge or made new holes in wooden nestboxes to access their content. ${ }^{49}$

Considering each individual component, moss was, not surprisingly, the most abundant material, amounting to $68-79 \%$ in the different species, and not differing significantly among them. Though this might seem obvious, knowing the general behaviour of these species, even this trait might show a great plasticity. For example, Álvarez et al. ${ }^{15}$ recorded that moss only amounted to $16 \%$ of the total mass of Great Tit nests in a population breeding in orange plantations in Eastern Spain. Concerning other materials, our results for Blue and Great tits broadly agree with previous studies $^{24,34}$ : Blue Tits generally make greater use of bark and feathers, while Great Tits use relatively less feathers and more hair. We are not aware of any detailed study on the use of nest materials by Coal Tits, so the present contribution is the first offering a thorough description. As a general observation, Perrins ${ }^{34}$ reported that Coal Tits, like Great Tits, rarely use feathers in the construction of their nests, a pattern also found in our studied population. As an outstanding characteristic of Coal Tit nests, when compared with the other two tit species, at least in this study area, is the disproportionate use of fluff, woolly materials from animal and artificial sources such as fur or thread, which amounts to an average of $21 \%$ of the total dry mass of the nest, and could reach up to $37 \%$ in individual nests.

When grouping the nest materials attending to their main assumed function, we found that all the three species use similar amounts of structural materials. Britt and Deeming ${ }^{24}$ found that Blue Tits include more structuring materials in their nests than Great Tits, and suggested that this difference could be due the use of the structuring materials by the smaller species to fill the bottom of the nestbox before it can make and line a nest cup. This obviously does not explain our results, where even Coal Tits, smaller than Blue Tits, use a similar amount of structural materials than Great Tits. Perhaps the relationship proposed by Britt and Deeming ${ }^{24}$ applies up to a certain nest mass but, once the need of covering the bottom of the nest has been adequately fulfilled, further increases of structural materials would depend on other factors not so strongly dependent on body size. For example, moss highly contributes to the regulation of the humidity of the nest, ${ }^{12}$ and local climatological conditions might similarly affect to all the breeding species.

Our results show that Coal Tits, the smaller species, made greater use of thermoregulatory materials than the other two species. However, no significant differences in the quantity of thermoregulatory materials was found between Great and Blue tits. This seems to contradict the 
hypothesis relating the body size with the insulating properties of the nest, as the size of Blue Tits is much smaller than that of Great Tits and closer to Coal Tits. However, as previously stated, this classification in "structural" and "thermoregulatory" materials is not exclusive. Deeming et al. ${ }^{39}$ have recently shown that the insulating properties of dry grass do not differ from that of animal hair and moss, with known thermoregulatory function. ${ }^{5,12}$ In addition, bark is known for, among other functions, provide thermal protection. ${ }^{50}$ We suggest that the sum of these materials of plant origin may be contributing to the thermal insulation of Blue Tits nests, which would result in better insulated nests than those of Great Tits, supporting the hypothesis relating body size and nest insulation.

Since environmental conditions were the same for all the species, differences in the use of different materials found in the present study should be attributed to species-specific preferences, either derived from physical (e.g. body size) or behavioural traits (e.g. risk perception). Some of the differences seem quite straightforward, as the greater use of fluff by Coal Tits, the smallest, and probably more sensible to cold, species, which is probably linked to its thermoregulatory properties. ${ }^{39}$ However, it is also noteworthy that the different species might use different strategies to face the same problem. Thus, while Coal Tits make frequent use of fluff, feathers seem to provide an alternative source of insulating material for Blue Tits. ${ }^{10,38,39}$ Similarly, Blue and Coal tits seem to prefer bark to compact the nest, while Great Tits used long and rigid wild boar hairs to satisfy this need.

Some nest materials might be present at the nest for several reasons other than thermoregulation and structuring. ${ }^{31}$ The use of aromatic plants with sanitary purposes has been described in several species including Blue and Great Tits. ${ }^{19,35,51}$ Feathers, apart from their insulating properties, seem to play an important role as a male quality signal in Blue Tit nests. ${ }^{13,35}$ This phenomenon has not been described to date in the other two tit species, so the greater use of feathers in the Blue Tits nests observed in our study could be partly a consequence of its function as quality signals in this species. As feathers potentially brought to decorate the nests would be later incorporated into the upper layer of the nest, probably mixing with the truly thermoregulatory material, it is not possible to assign how much of the feather mass was originally performing each function when deconstructing nests after fledging.

In conclusion, our results support that smaller species, probably more vulnerable to cold, build more insulating nests through making them bigger and/or by using more materials with thermoregulatory properties. Perceived predation risk might also contribute to the negative relationship between nest mass and mean body mass of the species. The existence of a differential selection of the available materials between species results in species-specific characteristics in the composition of nests. Thus, actually measuring the insulation properties of the nests, ${ }^{52}$ ideally before hatching, would be needed to properly test the thermoregulation hypothesis.

\section{Acknowledgements}

E.B. and E.A. conceived the study. E.B., D.D.-M. and J.V. conducted the fieldwork and collected the nests. I.A. deconstructed the nests. E. A. supervised nest deconstruction and helped with weighing. I.A. and D.D.-M. performed the statistical analyses. I. A. and E.B. drafted the manuscript, with inputs from E.A. and D.D.-M. All authors read and approved the manuscript. 


\section{Declaration of conflicting interests}

The authors declared no potential conflicts of interest with respect to the research, authorship or publication of this article.

\section{Funding}

This study was supported by projects CGL2013-48001-C2-1-P and CGL2016-79568-C3-1-P (Spanish Ministry of Economy and Competitiveness, MINECO). DD-M benefitted from an FPI fellowship (BES-2014-069191) provided by the MINECO, and was supported by the grant BABE 805189 (European Research Council) during the last stages of the project.

\section{References}

1. Hansell M. Bird nests and construction behaviour. Cambridge: Cambridge University Press, 2000.

2. Deeming DC. Effects of female body size and phylogeny on avian nest dimensions. Avian Biol Res 2013; 6: 1-11.

3. Mainwaring MC, Hartley IR, Lambrechts MM. et al. The design and function of birds' nests. Ecol Evol 2014; 4: 3909-3928.

4. Deeming DC, Mainwaring MC, Hartley IR, et al. Local temperature and not latitude determines the design of Blue Tit and Great Tit nests. Avian Biol Res 2012; 5: 203-208.

5. Mainwaring $\mathrm{MC}$, Deeming $\mathrm{DC}$, Jones $\mathrm{Cl}$. et al. Adaptive latitudinal variation in Common Blackbird Turdus merula nest characteristics. Ecol Evol 2014; 4: 851-861.

6. Edwards SC, Shoot TT, Martin RF, et al. It's not all about temperature: breeding success also affects nest design. Behav Ecol 2020; araa052

7. Mainwaring MC and Hartley IR. The energetic costs of nest building in birds. Avian Biol Res 2013; 6: 12-17.

8. Jelínek V, Požgayová $\mathrm{M}$, Honza $\mathrm{M}$, et al. Nests as an extended phenotype signal of female quality in the great reed warbler. J Avian Biol 2016; 47: 428-437.

9. Møller AP, Adriaensen F, Artemyev A, et al. Variation in clutch size in relation to nest size in birds. Ecol Evol 2014; 4: 3583-3595.

10. Møller AP. Egg predation as a selective factor for nest design: an experiment. Oikos 1987; 50: 91-94.

11. Álvarez $E$ and Barba $E$. Nest quality in relation to adult bird condition and its impact on reproduction in Great Tits Parus major. Acta Ornitho 2008; 43: 3-9

12. Mertens JAL. Thermal conditions for successful breeding in Great Tits (Parus major L.). Oecologia 1977; 28: 1-29.

13. Sanz JJ and García-Navas V. Nest ornamentation in blue tits: is feather carrying ability a male status signal? Behav Ecol 2011; 22: 240-247.

14. Lombardo MP, Bosman RM, Faro CA, et al. Effect of feathers as nest insulation on incubation behaviour and reproductive performance of tree swallows (Tachycineta bicolor). Auk 1995; 112: 973-981. 
15. Álvarez E, Belda EJ, Verdejo J, et al. Variation in Great Tit nest mass and composition and its breeding consequences: a comparative study in four Mediterranean habitats. Avian Biol Res 2013; 6: 39-46.

16. Alabrudzińska JM, Kaliński A, Słomczyński R, et al. Effects of nest characteristics on breeding success of great tits Parus major. Acta Ornitho 2003; 38: 151-154.

17. Møller AP. Nest predation selects for small nest size in the blackbird. Oikos 1990; 57: 237-240.

18. Tomás G, Merino S, Moreno J, et al. Nest weight and female health in the Blue Tit (Cyanistes caeruleus). Auk 2006; 123: 1013-1021.

19. Mennerat A, Mirleau P, Blondel J, et al. Aromatic plants in nests of the blue tit Cyanistes caeruleus protect chicks from bacteria. Oecologia 2009; 161: 849-855.

20. Holveck M-J, Grégoire A, Doutrelant C, et al. Nest height is affected by lamppost lighting proximity in addition to nestbox size in urban great tits. J Avian Biol 2019; 50: e01798

21. Sheldon FH and Winkler DW. Nest architecture and avian systematics. Auk 1999; 116: 875-877.

22. Lambrechts MM, Demeyrier V, Fargevieille A, et al. Great Tits build shallower nests than Blue Tits. Avian Biol Res 2014; 7: 251-254.

23. Winkler DW and Sheldon FH. Evolution of nest construction in swallows (Hirundinidae): A molecular phylogenetic perspective. Proc Natl Acad Sci 1993; 90: 5705-5707.

24. Britt $J$ and Deeming DC. First-egg date and air temperature affect nest construction in Blue Tits Cyanistes caeruleus, but not in Great Tits Parus major. Bird Study 2011; 58: 7889.

25. Lambrechts MM, Haurez J, Bodineau G, et al. Coal Tits Periparus ater build larger nests than Blue Tits Cyanistes caeruleus and Great Tits Parus major living in the same Mediterranean coniferous woodland habitat. Acta Ornithol 2016; 51: 123-129.

26. Smith JA, Harrison TJ, Martin GR, et al. Feathering the nest: food supplementation influences nest construction by Blue (Cyanistes caeruleus) and Great Tits (Parus major). Avian Biol Res 2013; 6: 18-25.

27. Kaliński A, Wawrzyniak J, Bańbura M, et al. Does the threat of European Pine Marten (Martes martes) predation influence the height of nests built by Blue Tits (Cyanistes caeruleus) and Great Tits (Parus major)? Avian Biol Res 2014; 7: 83-90.

28. Lambrechts MM, Blondel J, Dubuc-Messier G, et al. Great Tits build shallower nests than Blue Tits in an insular oak-dominated habitat mosaic. Avian Biol Res 2015; 8: 117-121.

29. Glądalski M, Bańbura M, Kaliński A, et al. Effects of nest characteristics on reproductive performance in Blue Tits Cyanistes caeruleus and Great Tits Parus major. Avian Biol Res 2016; 9: 37-43.

30. Navalpotro H, Pagani-Núñez E, Hernández-Gómez S, et al. Comparing prey composition and prey size delivered to nestlings by great tit, Parus major, and blue tit, Cyanistes caeruleus, in a Mediterranean sclerophyllous mixed forest. Anim Biodiver Conserv 2016; 39: 129-139.

31. Deeming DC and Mainwaring MM. Functional properties of nests. pp 29-49 in Deeming DC and Reynolds SJ (eds.) Nest, eggs, and incubation: New ideas about avian reproduction. Oxford Univ. Press, Oxford, 2015.

32. Tulp I, Schekkerman $\mathrm{H}$ and Leeuw J. Eggs in the freezer: energetic consequences of nest size and nest design in Artic breeding shorebirds. PLOS ONE 2012; 7: e38041.

33. Biddle LE, Broughton RE, Goodman AM, et al. Composition of bird nests is a speciesspecific characteristic. Avian Biol Res 2018; 11: 132-153. 
34. Perrins CM. British Tits. London: Collins, 1979.

35. Mainwaring MC. Causes and consequences of intraspecific variation in nesting behaviors: insights from Blue Tits and Great Tits. Front Ecol Evol 2017; 5: 39.

36. Álvarez $E$ and Barba E. ¿Cómo afecta la calidad del nido per se al proceso de incubación?: una aproximación experimental. Rev Cat Orn 2009; 25: 11-18.

37. McGowan A, Sharp SP and Hatchwell BJ. The structure and function of nests of Longtailed Tits Aegithalos caudatus. Funct Ecol 2004; 18: 578-583.

38. Pinowski J, Haman A, Jerzak L, et al. The thermal properties of some nests of the Eurasian tree sparrow Passer montanus. J Therm Biol 2006; 31: 573-581.

39. Deeming DC, Griffiths JD and Biddle LE. Material type and position determines the insulative properties of simulated nest walls. Ardeola 2020; 67: 127-136.

40. Maechler M, Rousseeuw P, Croux C, et al. robustbase: Basic Robust Statistics R package version 0.93-6. https://cran.r-project.org/web/packages/robustbase/index.html (2019, accessed 22 April 2020)

41. Zeileis A. Econometric Computing with $\mathrm{HC}$ and HAC Covariance Matrix Estimators. J Stat Softw 2004; 11: 1-17.

42. $\mathrm{R}$ Core Team. R: a language and environment for statistical computing. Vienna: $\mathrm{R}$ Foundation for Statistical Computing, www.r-project.org.

43. Fox, J. Effect displays in R for generalised linear models. J Stat Software 2003; 8: 1-27.

44. Minot EO and Perrins CM. Interspecific interference competition - nest sites for Blue and Great Tits. J Anim Ecol 1986; 55: 331-350.

45. Surgery J, Du Feu CR and Deeming DC. Opportunistic use of a wool-like artificial material as lining of tit (Paridae) nests. The Condor 2012; 114(2): 385-392.

46. Dickinson AM, Goodman AM and Deeming DC. Air movement affects insulatory values of nests constructed by Old World Warblers. J Therm Biol 2019; 81: 194-200.

47. McCleery RH, Clobert J, Juliard R, et al. Nest predation and delayed cost of reproduction in the great tit. J Anim Ecol 1996; 65: 96-104.

48. Miller KE. Nesting success of the Great Crested Flycatcher in nestboxes and in tree cavities: are nestboxes safer from nest predation? Wilson Bull 2002; 114: 179-185.

49. Skwarska JA, Kaliński A, Wawrzyniak J, et al. Opportunity makes a predator: Great Spotted Woodpecker predation on Tit broods depends on nestbox design. Ornis Fenn 2009; 86: 109-112.

50. Nicolai V. The bark of trees: thermal properties, microclimate and fauna. Oecologia 1986; 69: 148-160.

51. Mennerat $A$, Perret $P$, Bourgault $P$, et al. Aromatic plants in nests of blue tits: positive effects on nestlings. Anim Behav 2009; 77: 569574.

52. Cruz A, Álvarez E and Barba E. Nest insulating capacity during incubation and after fledging are related. Avian Biol Res 2016; 9: 22-27. 